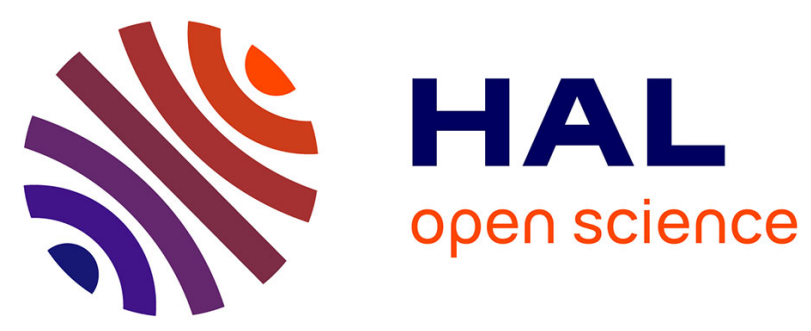

\title{
Harmonic mixing with Josephson point contacts
}

\author{
J.M. Lourtioz, R. Adde, G. Vernet, J.C. Henaux
}

\section{To cite this version:}

J.M. Lourtioz, R. Adde, G. Vernet, J.C. Henaux. Harmonic mixing with Josephson point contacts. Revue de Physique Appliquée, 1977, 12 (3), pp.487-492. 10.1051/rphysap:01977001203048700 . jpa00244201

\section{HAL Id: jpa-00244201 https://hal.science/jpa-00244201}

Submitted on 1 Jan 1977

HAL is a multi-disciplinary open access archive for the deposit and dissemination of scientific research documents, whether they are published or not. The documents may come from teaching and research institutions in France or abroad, or from public or private research centers.
L'archive ouverte pluridisciplinaire HAL, est destinée au dépôt et à la diffusion de documents scientifiques de niveau recherche, publiés ou non, émanant des établissements d'enseignement et de recherche français ou étrangers, des laboratoires publics ou privés. 


\author{
Classification \\ Physics Abstracts \\ 8.440
}

\title{
HARMONIC MIXING WITH JOSEPHSON POINT CONTACTS
}

\author{
J. M. LOURTIOZ, R. ADDE, G. VERNET et J. C. HENAUX \\ Institut d'Electronique Fondamentale ${ }^{*}$ ) \\ Bâtiment 220, Université Paris XI, 91405 Orsay, France
}

(Reçu le 2 juillet 1976, accepté le 6 décembre 1976)

\begin{abstract}
Résumé. - Des contacts ponctuels Josephson ont été utilisés pour effectuer la génération et le mélange d'harmoniques (jusqu'à 96) de klystrons microondes avec la radiation émise par un laser HCN à $891 \mathrm{GHz}$. Une analyse qualitative du mélange de deux radiations extérieures dans une jonction Josephson est effectuée et permet de concevoir l'existence de composantes de mélange indépendantes de la fréquence et de la largeur de raie de l'oscillation Josephson. L'analyse spectrale du laser HCN montre l'importance de la pureté spectrale de la source microonde lorsque des facteurs de multiplication élevés sont utilisés.

Abstract. - Experiments are described in which Josephson point contacts were used to generate and mix harmonics (up to the 96th) of microwave klystrons with the radiation from an HCN laser at $891 \mathrm{GHz}$. A qualitative analysis of mixing of two external radiations in a Josephson junction is given in order to understand the existence of mixing components independent of the Josephson oscillation frequency and linewidth. The spectral analysis of the HCN laser shows the importance of the microwave source spectral purity when frequency multiplication by large factors is considered.
\end{abstract}

1. Introduction. - An outstanding property of the Josephson junction as an harmonic mixer is its ability to convert an unusually large part of the power of a microwave incident signal (frequency $\omega_{1}$ ) into harmonics $\left(n \omega_{1}\right)$ of very high order and mix simultaneously in the device these harmonics with a farinfrared electromagnetic radiation (frequency $\omega_{2}$ ). The important result is the existence of mixing components at frequencies $\left|\omega_{2}-n \omega_{1}\right|$ independent of the Josephson oscillation frequency and linewidth which allows the possibility of performing highly precise frequency metrology of far infrared lasers as a function of a microwave frequency standard. The best performances which have been obtained are by Mc Donald et al. [1] who reported 401st order harmonic mixing of signals between a $9.5 \mathrm{GHz}$ klystron and a water vapor laser at $3.8 \mathrm{THz}$ and by T. G. Blaney and D. J. E. Knight [2] who observed 825th order harmonic mixing between a frequency multiplied signal at $1.08 \mathrm{GHz}$ and an $\mathrm{HCN}$ laser at $891 \mathrm{GHz}$.

The fact that there is a mixing component which is both independent of the Josephson frequency and linewidth has not yet been clearly explained and we give here a qualitative analysis of this characteristic. We report results [3,4] obtained concerning the spectral analysis of a stable free running $\mathrm{HCN}$ laser at

(*) Laboratoire associé au C. N. R. S.

REVUE de PHYSIQUe APPliQuéE. - T. 12, No 3, MARS 1977
$891 \mathrm{GHz}$ [5] with microwave references either at $9.3 \mathrm{GHz}(\times 96)$ or $34 \mathrm{GHz}(\times 26)$. Such an analysis has been done by Wells et al. [6] using the spectral density of the frequency fluctuations $S_{\delta v}$ of the microwave source, which implies a computer treatment. We have chosen to transpose at higher frequencies the recent analysis by Walls and Demarchi [7] based on the observation of the frequency spectrum $S_{v}$ of the microwave source. We discuss the results in view of experiments at a few teraherts and in particular the importance of the microwave reference spectral purity when frequency multiplication by large factors is considered.

2. The Josephson harmonic mixer. - When a Josephson junction is irradiated simultaneously at two frequencies $\omega_{1}$ and $\omega_{2}$, a simple voltage biased model [10] based on the ideal Josephson element predicts output current components at the frequencies

$$
\Omega=\left|\omega_{0}+l \omega_{1}+m \omega_{2}\right| \text {. }
$$

where $l$ and $m$ are integers (positive or negative), $\omega_{0}=\left(2 e V_{0} / \hbar\right)$ is the Josephson frequency and $V_{0}$ is the de voltage bias. The above relation involves only combinations of $\omega_{0}$ with $\omega_{1}$ and $\omega_{2}$ and consequently does not give beat signals independent of the Josephson oscillation. However, it has been found in the experiments [1-3] that beats were obtained which do not shift in frequency as $V_{0}$ is changed. Moreover 
the linewidths of the beat signals are also independent of the Josephson oscillation linewidth. The experiments therefore show that $\omega_{0}$ is not involved directly in these beat signals which may be written

$$
\Omega=\left|l \omega_{1}+m \omega_{2}\right|,
$$

The origin of this apparent discrepancy lies in the nature of the constant voltage biased model which does not apply generally to real Josephson junctions.

Most of the properties of superconducting point contacts (which are actually the best Josephson junctions at high frequencies), can be obtained by using the resistive shunted junction (RSJ) model [8] where an ideal Josephson element is shunted by a normal resistance $R_{\mathrm{N}}$ taking into account the current flow due to an imperfect dielectric and or metallic shorts as well as the tunneling electron current. Since $R_{\mathrm{N}}$ is usually small (a few ohms), a real junction must be considered as driven by constant current sources. If the junction is only driven by a constant dc source at $I>I_{\mathrm{c}}$, the feedback due to the voltage appearing across $R_{\mathrm{N}}$ results in a severe distortion of the Josephson oscillation which contains harmonics $p \omega_{0}$ of the fundamental frequency $\omega_{0}$ and a dc pair current flows in the contact which has been calculated analytically by Aslamazov and Larkin [8]. On the contrary, analytic solutions cannot be found for such an equivalent circuit fed simultaneously with dc and rf constant current sources at $\omega_{1}$ and $\omega_{2}$. However the mixing properties of the shunted junction have been determined by Hamilton [9] using analog computer simulation. He obtained a spectrum containing the combination frequencies

$$
\Omega=\left|p \omega_{0}+l \omega_{1}+m \omega_{2}\right| \text {. }
$$

The case $p=0$ corresponds to harmonic mixing experiments where the fundamental oscillation is not involved.

Thompson [11] has shown that this results from the mixing of the Josephson frequency $\omega_{0}$ and its harmonics $k \omega_{0}$ with the two applied frequencies at $\omega_{1}$ and $\omega_{2}$. He used a voltage source model taking feedback qualitatively into account in the junction and made a Fourier analysis. Eq. (1) must be replaced by

$$
\Omega=\left|\omega_{0}+k \omega_{0}+l \omega_{1}+m \omega_{2}\right|
$$

where

$$
p=k+1 \text {. }
$$

We consider now the effect of noise on the mixing current components of a Josephson junction mixer. The intrinsic noise of the junction does not have the same consequences on the different types of mixing components :

- Components $k \neq-1:$ In eq. (4), the frequency $\Omega$ depends explicitly of the fundamental frequency $\omega_{0}$ of the Josephson oscillation. Therefore these components are all affected by noise following the same mechanism which leads to the Josephson oscil- lation linewidth $\Delta \omega_{0}$ and their linewidth are related to $\Delta \omega_{0}$. It is well known thatt helatter is a consequence of the frequency modulation of the Josephson oscillation by low frequency noise components $[12,13]$. Since the Josephson frequency is very sensitive to the applied voltage at the junction $(2 e / h=484 \mathrm{MHz} / \mu \mathrm{V})$ the Josephson linewidths may be relatively very important [14, 15]. Consequently, an exact knowledge of the intrinsic noise level of the junction is required when the fundamental Josephson oscillation is used in a frequency converter.

- Components $k=-1$ : The corresponding mixing components are independent of the Josephson oscillation frequency and $\Omega=l \omega_{1}+m \omega_{2}$. It is shown by experience [1-3] and by an analogue computer simulation [9] that the linewidth of these components is independent of the Josephson oscillation linewidth $\Delta \omega_{0}$. When fluctuations are taken into account, since (i) only the low frequency ones are of importance and (ii) the Josephson junctions have a very high speed response so that the voltage and current in the junction are synchronous, the phenomenological analysis may be applied in a time scale of the order of the low frequency fluctuations. Therefore the Fourier analysis may still be used showing that the components $\Omega=l \omega_{1}+m \omega_{2}$ are completely independent of the Josephson oscillation and particularly that their linewidth is independent of $\Delta \omega_{0}$. The consequences of the intrinsic noise of the junction in the heterodyne mode with a local oscillator are then only related to the noise voltage across the junction and to the associated equivalent noise temperature at the mixer out put.

3. Apparatus (Fig. 1). - A free running hydrogen cyanide laser [5] provided the $891 \mathrm{GHz}$ signal. A high frequency stability and a sufficiently high output power are two requirements which are difficult ot match simultaneously in the case of the HCN laser. The laser was designed to satisfy both of the above conditions with emphasis on frequency stability using only passive techniques. It is characterized by a 6.5 meter semi-confocal cavity with temperature controled silica spacers. The radiation is coupled out with a Michelson interferometer configuration using a polyethylene beam splitter and identical plane mirrors at the beam splitter end of the cavity. The electrical discharge is operated in a completely steady striated regime from cathode to anode with $\mathrm{CH}_{4}-\mathrm{N}_{2}$ : 1-1 gas mixture and a stabilized current supply. A good thermalization of the plasma and good homogeneity of the discharge is obtained by the addition of helium. The total gas pressure is $\sim 0.4$ torr. The laser gives a $20-30 \mathrm{~mW}$ power output in the best frequency stability conditions when the fractional current fluctuations are $\lesssim 10^{-3}$.

The microwave signal is given by a klystron thermostated in an oil bath and stabilized from a $5 \mathrm{MHz}$ quartz crystal with a MOS-5 (Microwave System) 




FIG. 1. - Apparatus used to investigate harmonic mixing with the HCN laser.

synchronizer. Two types of klystrons were used oscillating respectively in $\mathrm{X}$-band $(\simeq 9.3 \mathrm{GHz})$ and $\mathrm{K}$ band $(\simeq 34.2 \mathrm{GHz})$.

An optimized detection of the mixing current component at the intermediate frequency

$$
\omega_{\text {i.f. }}=\left|n \omega_{1}-\omega_{2}\right|
$$

implies the need to match simultaneously the Josephson point contact with the infra-red radiation at $\omega_{2}$, the microwave signal at $\omega_{1}$ and the amplifier chain at $\omega_{\text {i.f. }}$. This is a rather difficult task due to the very different frequencies involved and the low impedance of Josephson junctions.

The superconducting point contact consists of a sharpened $2 \mathrm{~mm}$ diameter $\mathrm{Nb}$ rod pressed on a flat $\mathrm{Nb}$ plate with a precise pressure adjusting mechanism [15]. Both pieces are machined from bulk metal and the surfaces are first mechanically polished then chemically etched for a few seconds in the following solution $\left(60 \% \mathrm{HF}, 30 \% \mathrm{HNO}_{3}, 10 \%\right.$ $\mathrm{H}_{2} \mathrm{O}$ ). The cone angle $2 \theta$ is generally $\sim 120^{\circ}$ and the radius of curvature at the point is $\sim 5 \mu \mathrm{m}$. This structure makes the contact operate in the far-infrared as a conical antenna [16] with dimensions a few wavelengths transversely while the contact area is much less than the wavelength. The coupling of the external radiation to the Josephson device may be viewed in the following way. The low impedance point contact (a few $\Omega$ ) must be adapted to the external free space impedance. Due to the spherical shape of the conical $\mathrm{Nb}$ rod near the tip, the structure is equivalent in the immediate neighbourhood of the contact to a conical antenna with a very large angle (2 $\theta$ near $180^{\circ}$ ) and has a corresponding very low radiative impedance (a few $\Omega$ ) [16] allowing matching with the Josephson point contact. Moreover the spherical shape in the contact area gives a tapered transition and matching to the main cone $\left(120^{\circ}\right)$ which angle gives broadband properties to this type of antenna.

The $\mathrm{X}$ band waveguide containing the $\mathrm{Nb}-\mathrm{Nb}$ contact is mounted in a sealed stainless tube in contact with the external liquid helium bath. The contact is cooled both by conduction with its mount and convection of the exchange gas at a slighly reduced pressure. This prevents noise from liquid helium bubbling and provides simultaneously an electric shield. The $\mathrm{Nb}-\mathrm{Nb}$ contact is biased with a $\mathrm{Hg}$ battery and a potentiometric system. Filters with time constants of the order of 0.1 second are necessary on the four leads inside the cryostat to eliminate the effect of external noise sources and especially the $50 \mathrm{~Hz}$. The infrared radiation is fed through the $X$ band waveguide from the top of the cryostat with a first focussing mirror to minimize losses in the waveguide. The sliding short is machined as a spherical mirror $(R=1 \mathrm{~cm})$ to match the $\mathrm{X}$ band radiation and simultaneously focus the $891 \mathrm{GHz}$ radiation on the 
contact. The i.f. selected frequency is $110 \mathrm{MHz}$. Matching between the point contact and the i.f. receiver is performed with a resonant circuit at $110 \mathrm{MHz}$ with $Q \sim 30$.

Two methods were used to visualize the beat signals. In oscilloscope video measurements the klystron is unlocked and frequency swept with a very small amplitude. Therefore the i.f. mixing signal is also frequency swept and after detection and amplification in the $110 \mathrm{MHz}$ receiver which passband may be varied $(300 \mathrm{kHz}, 20 \mathrm{kHz})$, is visualized on an oscilloscope. Consequently the signal is observed in a window with fixed frequency and selected width. This method is always used in preliminary experiments to optimize the i.f. signal as a function of the different variable parameters (junction characteristic and operating point, applied radiation powers, mirrors orientations). However there is a minimum frequency (a few $10^{2}$ hertz) necessary to modulate the klystron power supply and this method only allows observations of the signal with $\tau \leqslant 10^{-3}$ second. In spectrum analyzer measurements the klystron is operated at a fixed frequency by locking on a MOS-5 synchronizer. The signal observation time may be varied from a few $10^{-1}$ seconds to $10^{2}$ seconds.

4. Experimental results and discussion. - Several features of the junctions appeared desirable in the course of the experiments in order to achieve good harmonic mixer operation. They are mainly related to the optimization of their frequency properties in the submillimeter and far infrared region and confirm other observations $[1,2]$. The normal resistance $R_{\mathrm{N}}$ of the junction is responsible of a first limitation which is related to a time constant

$$
\tau_{\mathrm{c}}=f_{\mathrm{c}}^{-1}=\left[(2 e / h) R_{\mathrm{N}} I_{\mathrm{c}}\right]^{-1} .
$$

The junction used in the experiments have normal resistances $R_{\mathrm{N}}$ of a few ohms and critical currents $\sim 100 \mu \mathrm{A}$. The values of $R_{\mathrm{N}} I_{\mathrm{c}}$ are in the range $0.1-0.5 \mathrm{mV}$, and are typical of those encountered in the literature. However they are far from the theoretical value (calculated for tunnel junctions) $\pi \Delta / 2 \simeq 2.2 \mathrm{mV}$ for niobium at $4.2 \mathrm{~K}$ which corresponds to $f_{\mathrm{c}} \simeq 10^{12} \mathrm{~Hz}$. Such values seem convenient for experiments in the frequency region $\lesssim 1 \mathrm{THz}$, but it should be desirable at higher frequency to reach the maximum theoretical value of $R_{\mathrm{N}} I_{\mathrm{c}}$. Another limitation at very high frequencies is due to the shunt capacitance of the junction which is related to another time constant

$$
\tau_{\mathrm{c}}^{\prime}=2 \pi\left(\hbar C / 2 e I_{\mathrm{c}}\right)^{1 / 2} .
$$

For example, with $I_{\mathrm{c}}=100 \mu \mathrm{A}$ and $C \sim 0.1 \mathrm{pF}$, $f_{\mathrm{c}}^{\prime}=\left(\tau_{\mathrm{c}}^{\prime}\right)^{-1} \sim 310^{11} \mathrm{~Hz}$, which means that small area point contacts have to be realized. This was obtained with contacts having small radius of curvature $(\sim 5 \mu)$ and a very light pressure adjustment.
Finally, the possibility of thermal effects cannot be neglected at these very high frequencies, in spite of the relatively massive structure of the superconducting electrodes forming the point contact.

Beat signals at the i.f. where always obtained with contacts showing an observable first induced step on the VI characteristic corresponding to the laser frequency. The ratio of the signal amplitude to the noise amplitude was currently $\gtrsim 30 \mathrm{~dB}$. Figure 2

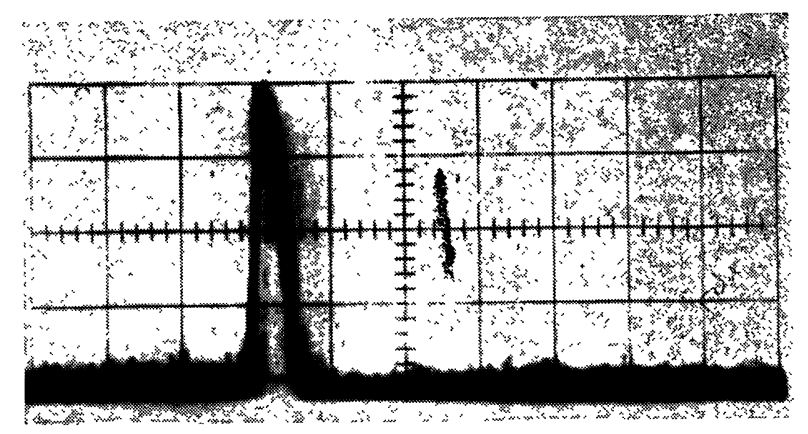

(a)

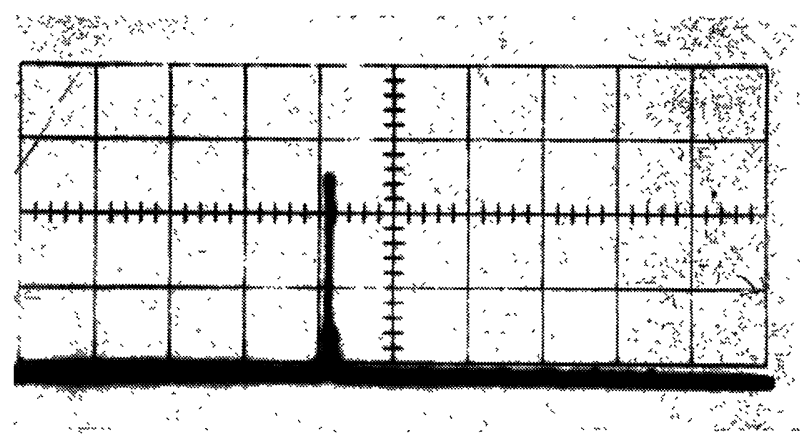

(b)

Fig. 2. - Video observation of the beat signal beetween the 9.3 GHz klystron $(\times 96)$ slightly frequency modulated and the HCN laser (891 GHz). In (A) and (B) the vertical and horizontal scales are both linear (horizontal calibration : $1 \mathrm{MHz} / \mathrm{div}$ ). In (A) the receiver window is $300 \mathrm{kHz}$, in (B) $20 \mathrm{kHz}$.

gives a typical example of the results obtained in video detection. They correspond to the beat signal between the HCN laser at $891 \mathrm{GHz}$ and the 96th harmonic of the $9.3 \mathrm{GHz}$ klystron weakly frequency modulated (a few $10^{2} \mathrm{~Hz}$ ). In that case the detected signal linewidth is equal to the receiver window (case A : $300 \mathrm{kHz}$, case $\mathrm{B}: 20 \mathrm{kHz}$ ) which shows that the beat signal has a width narrower than $20 \mathrm{kHz}$. Consequently the linewidth of the free running laser is less than $20 \mathrm{kHz}$ over time period $\tau \approx 10^{-3} \mathrm{~s}$. Similar results were obtained with the $34 \mathrm{GHz}$ klystron. From the klystron frequency measured with a $\mathrm{H}$. P. 5360 A counter the laser frequency measurements determined with this method are given by

$$
F=890.7605 \mathrm{GHz} \pm 300 \mathrm{kHz}
$$

and are mainly limited in precision by the laser cavity tuning on the centre of the emission line. 
The result of the spectral analysis of the beat signal when the microwave source (34 GHz klystron) is phase locked on the MOS 5 are shown in figure 4. All other experimental conditions are comparable. We observe that only a weak beat signal comes out of a broad intense pedestal. As the video detection shows that the laser linewidth is $<20 \mathrm{kHz}$, the present result is due to noise introduced by the frequency locked klystron which we now analyze.

Frequency multiplication by large factors $n$ imposes very severe requirements on the spectral purity of the r.f. source. The noise power in the sidebands of its frequency spectrum increases approximately as $n^{2}$ relatively to the signal power [17]. Therefore the r. f. source noise may become dominant in the frequency fluctuations of the mixing signal in an harmonic mixer. This point may be seen qualitatively as follows :

The signal of an r. f. oscillator can be represented by

$$
v_{1}(t)=v_{1} \exp j\left(2 \pi f_{0} t+\varphi(t)\right)
$$

where $\varphi(t)$ represents the phase variations following a stochastic process which is generally assumed to be gaussian, about the linearly progressing phase $2 \pi f_{0} t$. When the level of fluctuations is weak $\left(\overline{\varphi(t)^{2}} \ll 1\right)$ the r. f. signal frequency spectrum may be written [7]

$S_{\mathrm{v}}(f) \simeq \exp \left(-\overline{\varphi(t)^{2}}\right) \times\left[\delta\left(f-f_{0}\right)+S_{\varphi}\left(f-f_{0}\right)\right]$

where $S_{\varphi}(f)$ is the spectral density of the phase fluctuations. The corresponding experimental spectrum is constituted by a carrier $\delta\left(f-f_{0}\right)$ and a pedestal $S_{\varphi}\left(f-f_{0}\right)$. The relative noise power in the pedestal is

$$
P_{0}=\exp \left(\overline{\varphi(t)^{2}}\right)-1 \simeq \overline{\varphi(t)^{2}}
$$

Let us now multiply the frequency of the above signal by $n$, the instantaneous signal phase is also multiplied by $n$

$$
v_{n}(t)=v_{n} \exp j\left(2 \pi n f_{0} t+n \varphi(t)\right) .
$$

First if $\overline{(n \varphi(t))^{2}}$ is small due either to a small $n$ value or to a very low noise level $\overline{\varphi(t)^{2}}$ of the r. f. source, the eqs. (6) and (7) may be transposed directly to $v_{n}(t)$ in eq. (4),

$$
\begin{aligned}
S_{\mathrm{v}_{n}}(f) \simeq \exp (- & \left.n^{2} \overline{\varphi(t)^{2}}\right) \times \\
& \times\left[\delta\left(f-n f_{0}\right)+n^{2} S_{\varphi}\left(f-n f_{0}\right)\right] \\
P_{n} \simeq & \exp \left(n^{2} \overline{\varphi(t)^{2}}\right)-1 \simeq n^{2} \overline{\varphi(t)^{2}} .
\end{aligned}
$$

Equation (9) shows that the frequency spectrum of the frequency multiplied signal $v_{n}(t)$ is identical to the spectrum of the reference signal $v_{1}(t)$. Therefore, the linewidth of the pedestal is unchanged $\left(\Delta f\left(P_{n}\right)=\Delta f\left(P_{0}\right)\right)$. Eq. (10) shows that the relation power in the pedestal is multiplied by $n^{2}$.

When $n$ is very large (or if the reference source has a poor spectral purity), $n^{2} \overline{\varphi(t)^{2}}$ becomes important so that the relations (9) and (10) are no more strictly valid. However Walls and Demarchi [7] have shown experimentally by frequency multiplication from a $5 \mathrm{MHz}$ crystal up to $\mathrm{X}$ band at $9.1 \mathrm{GHz}$ that the first expression of eq. (10) still was an excellent approximation even for values of $n^{2} \overline{\varphi(t)^{2}}>1$. We can therefore write

$$
P_{n} \simeq \exp \left(P_{0} n^{2}\right)-1 .
$$

Eq. (9) shows that the noise level has now an exponential variation in $n^{2}$, which means that the increase in phase noise amplitude is still more dramatic. Walls and Demarchi have established experimentally in this latter case an evolution law giving the broadening of the pedestal for very large values of $n$.

The results obtained in the spectral analysis of the beat signal between a $34 \mathrm{GHz}$ klystron and an $\mathrm{HCN}$ laser bring a confirmation at a higher frequency of the experimental study by Walls and Demarchi. When the $34 \mathrm{GHz}$ klystron is locked with the MOS-5 synchronizer, the frequency spectrum of the klystron (Fig. 3) measured on a H. P. 8555 A spectrum analyser
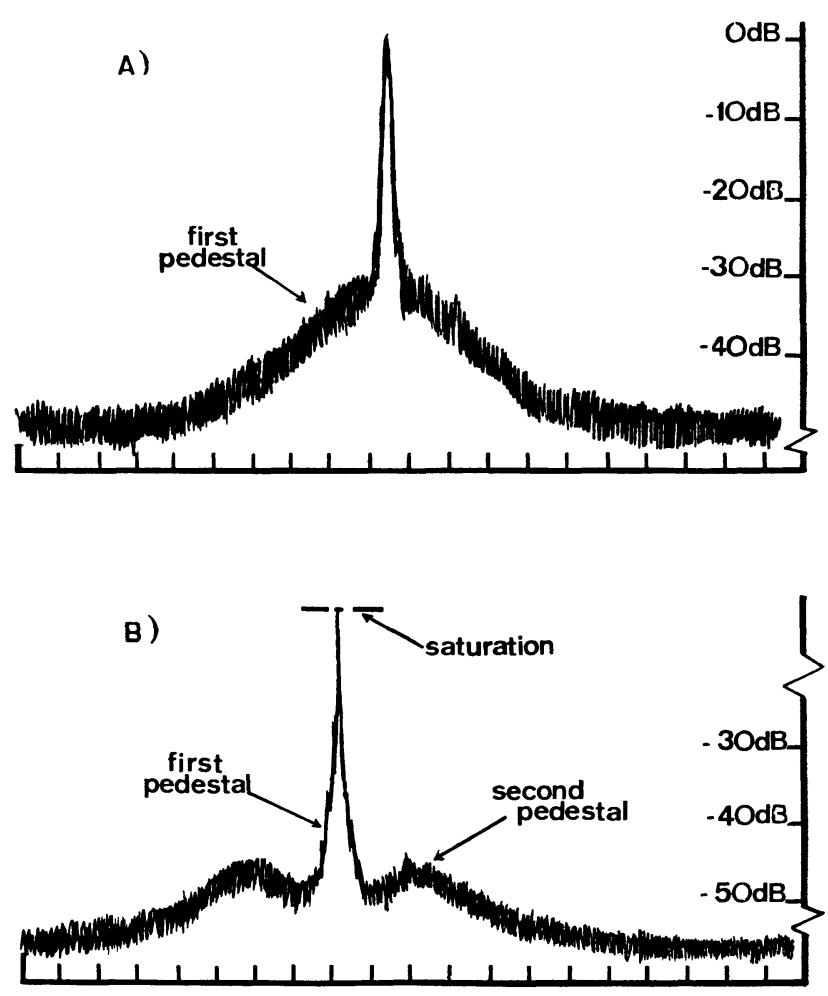

FIG. 3. - Spectrum of the $34 \mathrm{GHz}$ klystron phase locked on the synchronizer. In (A) and (B) the I. F. bandwidth of the spectrum analyser is $100 \mathrm{~Hz}$. The horizontal scale is $2.5 \mathrm{kHz} /$ div. in (A), $25 \mathrm{kHz} /$ div. in (B).

is given by a narrow intense peak (carrier) in the middle of two pedestals, the more intense peaking at $-30 \mathrm{~dB}$ with a $10 \mathrm{kHz}$ linewidth. The relative noise power in this pedestal is $\simeq 10^{-2}$. This type of spectrum does not correspond to a high purity type source and seems typical of the stabilization equipment which is continuously frequency tunable. As a consequence 
its short term stability is much less than could be obtained with a fixed frequency metrological quartz. From eq. (11) the relative estimated power in the pedestal after frequency multiplication $(n=26)$ is $P_{n} \simeq \exp (6.76)$ and owing to the relative linewidths of the signal and of the pedestal this means that the central peak is within the limits of observability after

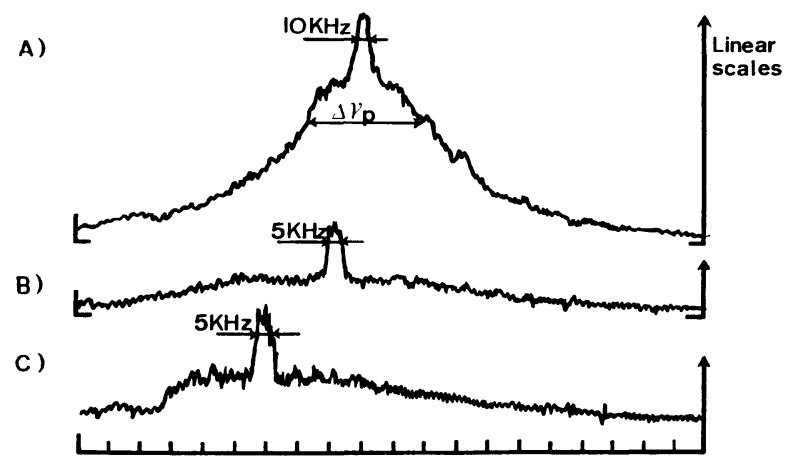

Fig. 4. - Spectrum of the beat signal beetween the $34 \mathrm{GHz}$ klystron phase locked on the MOS 5 stabilizer and the $\mathrm{HCN}$ laser showing the signal at the limit of detection from the pedestal due to the multiplication in the harmonic mixer of the phase noise of the klystron. In A) the horizontal scale is $25 \mathrm{kHz} / \mathrm{div}$., in B) and C) $10 \mathrm{kHz} / \mathrm{div}$. The I. F. bandwidths of the spectrum are respectively $10 \mathrm{kHz}, 3 \mathrm{kHz}, 1 \mathrm{kHz}$ in A), B) and C). The vertical scales (arbitrary units) are linear with respect to voltage. frequency multiplication. Moreover the experimental curve of Walls and Demarchi gives a pedestal linewidth $\Delta v_{n} \simeq 50-60 \mathrm{kHz}$.

The r. f. power spectrum of the beat signal between the $34 \mathrm{GHz}$ klystron frequency locked on the MOS-5 stabilizer and the HCN laser (891 GHz) confirms both of these predictions (Fig. 4), showing the signal coming out barely from the pedestal. When the same experiment is performed with the frequency locked $9 \mathrm{GHz}$ klystron $(n=96)$ the central peak is completely buried in a pedestal with a width $\simeq 300 \mathrm{kHz}$.

5. Conclusion. - The qualitative analysis of the mixing process in a Josephson junction which we have given allows one to understand fairly well the origin of the beat signals independent of the Josephson frequency fluctuations which make this device most competitive as an harmonic mixer for frequency metrology in the far-infrared. The experimental results obtained in the spectral analysis of an $\mathrm{HCN}$ laser show the importance of a high spectral purity of the microwave source when frequency multiplication by large factors is considered. Microwave solid state oscillators or klystrons stabilized on a high $Q$ superconducting cavity [18] should allow one to overcome this difficulty up to the $10 \mathrm{THz}$ region and are now under development in the laboratory.

\section{References}

[1] Mc Donald, D. G., Risley, A. S., Cupp, J. D., Evenson, K. M. and Ashley, J. R., Appl. Phys. Lett. 20 (1972) 296.

[2] Blaney, T. G. and KNight, D. J. E., J. Phys. D 7 (1974) 1882.

[3] LouRTioz, J. M., Thèse Docteur-Ingénieur, Orsay (1975) unpublished.

[4] Lourtioz, J. M., Adde, R., Vernet, G. and Hénaux, J. C., Journées Nationales Microondes, Limoges, 10-12 mars 1976.

[5] Lourtioz, J. M. and AdDe, R., Revue Phys. Appl. 11 (1976) 533.

[6] Wells, J. S., Mc Donald, Risley, A. S., JaRvis, S. and Cupp, J. D., Revue Phys. Appl. 9 (1974) 285.

[7] Walls, F. L. and Demarchi, A., IEEE IM24 (1975) 210.
[8] Aslamazov, L. G. and Larkin, A. I., Sov. Phys. JeTP Lett. 9 (1969) 87.

[9] Hamilton, C. A., J. Appl. Phys. 44 (1973) 2371.

[10] Grimes, C. C. and Shapiro, S., Phys. Rev. 169 (1968) 397.

[11] Thompson, J. Appl. Phys. 44 (1973) 3310.

[12] Stephen, M. J., Phys. Rev. 182 (1969) 531.

[13] Dahm, J., Denenstein, A., Langenberg, D. N., ParKER, W. H., Rogovin, D. and Scalapino, D. J., Phys. Rev. Lett. 22 (1969) 1416.

[14] Vernet, G. and Adde, R., Appl. Phys. Lett. 19 (1971) 195.

[15] Vernet, G. and Adde, R., J. Appl. Phys. 45 (1974) 2678.

[16] JASIK, H., Antenna Engineering Handbook (Mc Graw Hill, New-York) 1961.

[17] Middleton, D., Introduction to statistical Communication (Mc Graw-Hill, New-York) 1960.

[18] JimENEZ, J. J., Thèse Doctorat, Orsay, (1974) unpublished. 